\title{
Influence of the input-stage architecture on the in-laboratory test of a mid-infrared interferometer: application to the ALOHA up-conversion interferometer in the $L$ band
}

\author{
Julie Magri ${ }^{\circledR},{ }^{1 \star}$ Lucien Lehmann ${ }^{\circledR},{ }^{1}$ Ludovic Grossard, ${ }^{1}$ Laurent Delage, ${ }^{1}$ François Reynaud,${ }^{1 \star}$ \\ Mathieu Chauvet, ${ }^{2}$ Florent Bassignot, ${ }^{3}$ Rodolphe Krawczyk ${ }^{4}$ and Jean-Michel Le Duigou ${ }^{5}$ \\ ${ }^{1}$ Université de Limoges, CNRS, XLIM, UMR 7252, F-87000 Limoges, France \\ ${ }^{2}$ FEMTO-ST Institute, UMR CNRS 6174, Université de Bourgogne Franche-Comté, F-25000 Besançon, France \\ ${ }^{3}$ Femto-Engineering, $15 B$ avenue des Montboucons, F-25000 Besançon, France \\ ${ }^{4}$ Thales Alenia Space, Observation Exploration \& Navigation, 5 Allée des Gabians BP 99, F-06156 Cannes La Bocca Cedex, France \\ ${ }^{5}$ CNES, Centre Spatial de Toulouse, Service DSO/SI/OP, 18 avenue Edouard Belin, F-31401 Toulouse Cedex 09, France
}

Accepted 2020 October 19. Received 2020 September 28; in original form 2020 July 6

\begin{abstract}
In the framework of the Astronomical Light Optical Hybrid Analysis (ALOHA) laboratory mid-infrared (MIR) up-conversion fibred interferometer in the $L$ band, we report on the influence of the input-stage architecture. Using an amplitude division set-up in the visible or near-infrared is a straightforward choice in most cases. In the MIR context, the results are slightly different and we show that a wavefront division set-up is needed. These in-laboratory principle experiments allow us to measure a reliable 88 per cent instrumental contrast with high flux and to obtain fringes from faint sources at $3.5 \mu \mathrm{m}$ with a spectral bandwith of $37 \mathrm{~nm}$ converted to $817 \mathrm{~nm}$. An equivalent limiting $L$-band magnitude around 3.9, equivalent to $3.0 \mathrm{fW} \mathrm{nm}^{-1}$, could be demonstrated on $1 \mathrm{~m}$ class telescopes. This opens the possibility of planning future on-sky tests at the Center for High Angular Resolution Astronomy (CHARA) array and of predicting the performance attained.
\end{abstract}

Key words: instrumentation: high angular resolution-techniques: interferometric.

\section{INTRODUCTION}

The mid-infrared (MIR) spectral domain arouses huge interest in the observation of active galactic nuclei, young stellar objects, and the formation or evolution of planetary systems, but it requires very high angular resolution in the range of milliarcseconds. In order to obtain these characteristics, high-resolution imaging is used in astronomy through the mutual coherence measurement of electric fields collected by a telescope array (Lawson 2000). In this framework, MATISSE (Lopez et al. 2014), which operates in the Very Large Telescope Interferometer (VLTI), produces data in the $L, M$, and $N$ bands. The sensitivity of this interferometric instrument is drastically impacted by the thermal background radiated by the instrument itself and it therefore requires a complex cryogenic system. As shown in Fig. 1, the ALOHA (Astronomical Light Optical Hybrid Analysis) project proposes an alternative solution using an up-conversion interferometer to limit the impact of the thermal noise contribution and takes advantage of a long baseline using optical fibres (Lehmann et al. 2018). The up-conversion stages at the focus of each telescope shift the astronomical light from the MIR to the near-infrared (NIR) by using a sum-frequency generation (SFG) process (Boyd 1977) in a non-linear crystal. Validation of the principles of this concept was performed in 2008 (Brustlein et al. 2008) with widely available components dedicated to the telecom window $(1.55 \mu \mathrm{m}$, i.e. the $H$ band). After in-laboratory demonstrations (Gomes et al. 2014), on-

^E-mails: julie.magri@xlim.fr (JM); francois.reynaud@unilim.fr (FR) sky results were obtained in 2015 (Darré et al. 2016) in collaboration with the Center for High Angular Resolution Astronomy (CHARA) array team (Brummelaar et al. 2005). Since these successful results in the $H$ band $\left(H_{\text {mag }}=3.0\right)$, we have focused our work on the application of this new kind of instrument in the $L$ band to reach the MIR. For this purpose, in-laboratory experiments have been performed to prepare the following on-sky demonstration. In 2016, interference fringes were obtained in the laboratory with an attenuated laser monochromatic coherent source at $3.39 \mu \mathrm{m}$ (Szemendera et al. 2016). In 2019, a real on-sky sensitivity test using a single arm of the future ALOHA up-conversion interferometer at $3.5 \mu \mathrm{m}$ demonstrated the potential of the ALOHA project in the $L$ band. We detected stars with magnitudes as low as $L_{\mathrm{mag}}=2.8$ despite a low coupling efficiency (Lehmann et al. 2019b).

The goal of this paper is to explain why a wavefront division set-up is a key point of this architecture. It also reports on the current performances of the ALOHA in-laboratory experimental setup in order to prepare the future on-sky experiment at the CHARA array. For this purpose, we will focus on the differences between the amplitude and wavefront division experimental set-ups, the data processing, the experimental results, and the prospects of the upconversion interferometer in the $L$ band at $3.5 \mu \mathrm{m}$.

\section{LABORATORY EXPERIMENTAL SET-UP}

Fig. 2 gives a schematic view of the laboratory mid-infrared upconversion interferometer. It is composed of three main stages: 


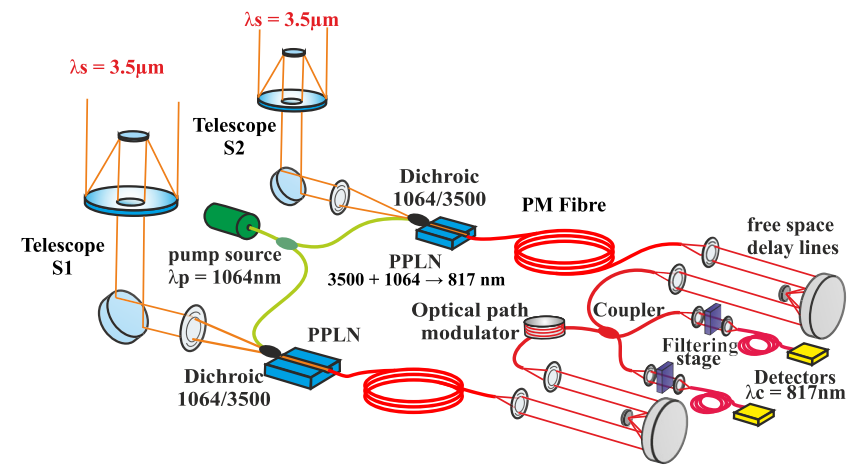

Figure 1. General principle of ALOHA. The optical fields collected by the two CHARA telescopes S1 and S2 are frequency shifted in non-linear waveguides (periodically poled lithium niobate; PPLN) from $3.5 \mu \mathrm{m}$ to $817 \mathrm{~nm}$ with the help of a $1064 \mathrm{~nm}$ laser pump. The beams are then transported through polarization-maintaining (PM) optical fibres to the CHARA freespace delay lines. Then, the two converted beams are mixed in a fibre coupler and the fringe pattern is detected in the time domain by two silicon photoncounting detectors. Notice that, with the telescope configuration, interferences are obtained by wavefront division.

(i) a $3.5 \mu \mathrm{m}$ stage that mimics the light coming from the astronomical target and splits it into the two interferometric arms;

(ii) an up-conversion stage shifting the spectrum from the MIR to the NIR at $817 \mathrm{~nm}$ using sum-frequency generation (SFG);

(iii) an $817 \mathrm{~nm}$ stage with optical field interferometric mixing.

In the following part of this paper, $\lambda_{s}$ and $\lambda_{p}$ correspond to the MIR signal and pump wavelengths, respectively, and $\lambda_{\mathrm{c}}$ is the converted NIR signal.

The MIR stage consists of a thermal source spatially filtered through a single-mode fluoride glass fibre. The spatial filtering performed by the single-mode waveguide guarantees the full spatial mutual coherence of the beams injected into the two arms of our instrument. The optical field at the output of the fluoride fibre is shared to the two arms by a beamsplitter.

In order to mimic the large range of flux encountered in the real onsky configuration, we used a thermal source, whose intensity emitted per spatial mode is theoretically determined through the blackbody Planck's law. A copper plate is thermally controlled by a Peltier thermoelectric cooler module. This plate is coated with a thin layer of candle soot with an estimated emissivity greater than 90 per cent. A combination of a $\mathrm{CaF}_{2}$ lens and an $L$-band filter allows the injection of the source into the fluoride glass fibre and removes any radiation in the vicinity of $800 \mathrm{~nm}$.

In the SFG stage, the optical field provided by a continuous-wave pump laser at $1064 \mathrm{~nm}$ is split into the two arms of the interferometer by a single-mode polarization-maintaining fibre coupler. In each arm, the MIR signal is mixed with the $1064 \mathrm{~nm}$ pump field thanks to a $\mathrm{ZnSe}$ dichroic mirror with high reflection at $1064 \mathrm{~nm}$ and antireflection at $3.5 \mu \mathrm{m}$ coatings. An off-axis parabola injects both the $3.5 \mu \mathrm{m}$ and the $1064 \mathrm{~nm}$ pump radiation into a PPLN ridge waveguide where sumfrequency generation takes place to give rise to a $817 \mathrm{~nm}$ converted optical field. The PPLN ridge waveguides were manufactured by Femto-Engineering (Chauvet et al. 2016; Lehmann et al. 2019c). The waveguide design parameters were chosen to comply with the signal and pump wavelengths. The two waveguides used in our experiment are $20 \mathrm{~mm}$ long, $7.5 \mu \mathrm{m}$ high, and, respectively, 7 and $8 \mu \mathrm{m}$ wide. Their poling periods are 19.6 and $19.9 \mu \mathrm{m}$, respectively, and the temperature of each waveguide is independently adjusted close to ambient temperature ( 16.5 and $19.5^{\circ} \mathrm{C}$, respectively). These temperature values are set to reach quasi-phase matching at the very same working wavelengths for both PPLN components. Weak discrepancies between the two PPLN characteristics remain due to small opto-geometrical differences between the two waveguides.

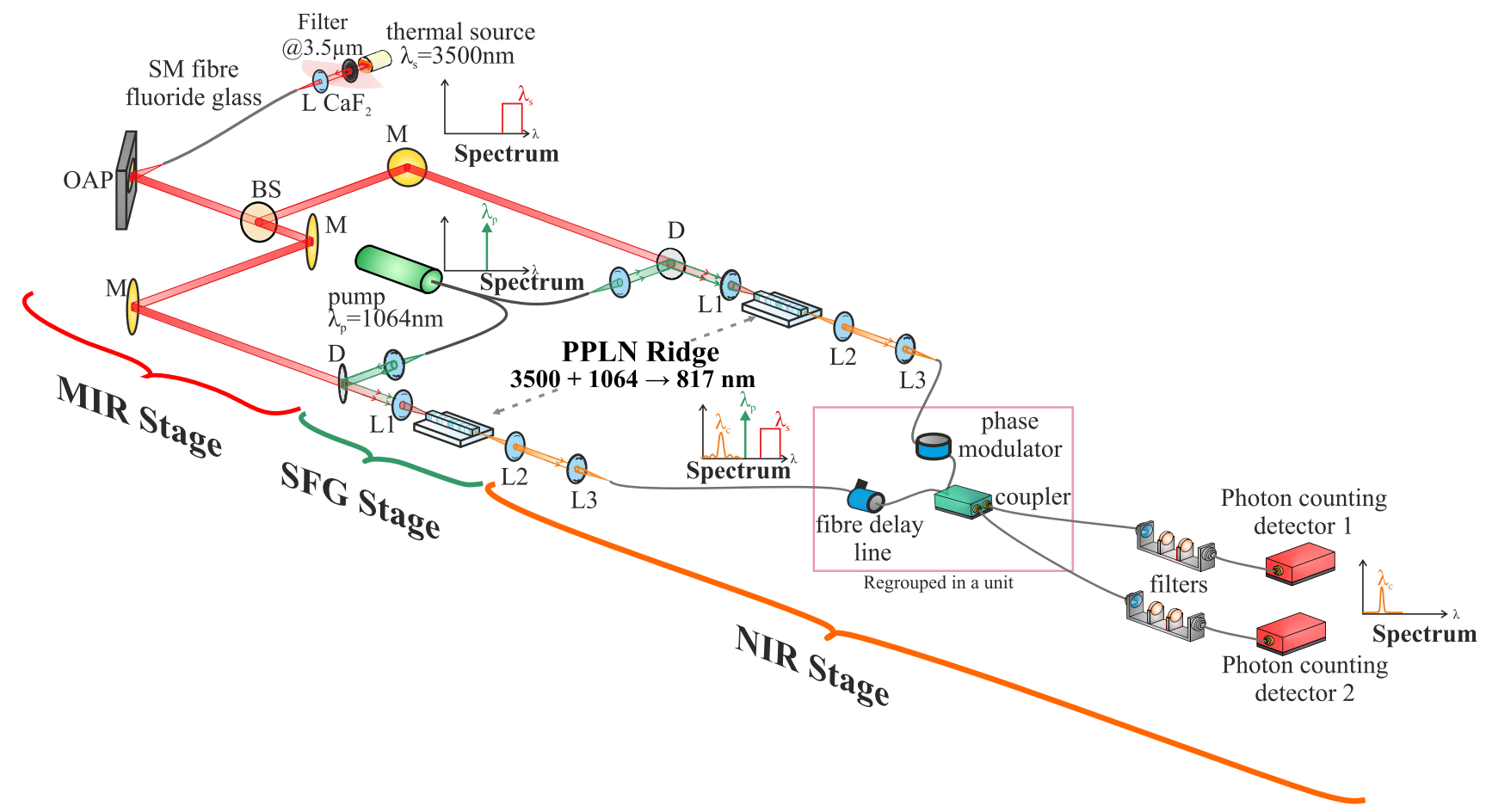

Figure 2. Diagram of the interferometric bench. $\mathrm{L} \mathrm{CaF}_{2}: \mathrm{CaF}_{2}$ lens. OAP: off-axis parabola. D: dichroic mirror (HR@1064 nm and AR@3.5 $\mu$ m). L1: off-axis parabola (a lens is shown for visibility reasons). L2, L3: microscope objectives. M: mirror. BS: beamsplitter. 


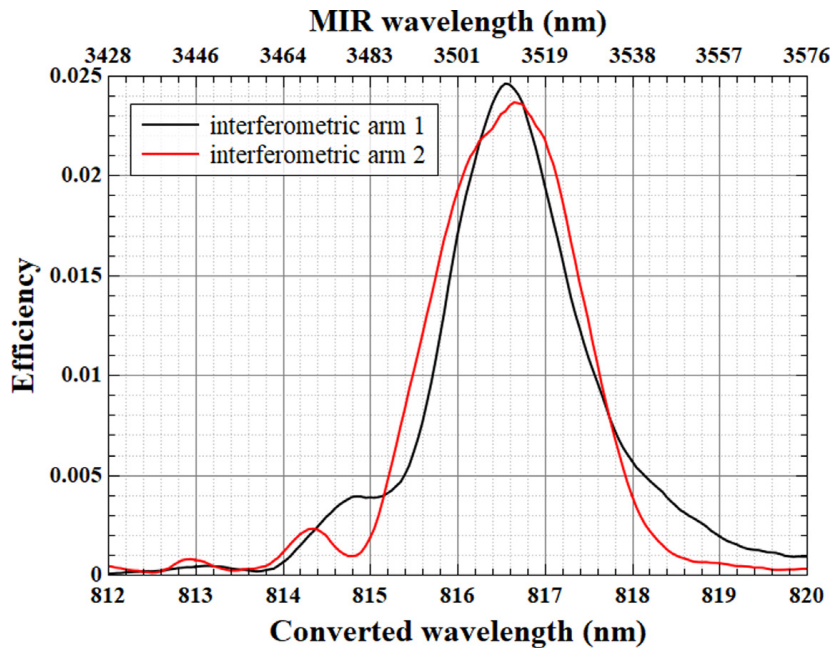

Figure 3. Spectral acceptances of the two waveguides used for the interferometric measurements.

This is depicted in Fig. 3 where the spectral acceptances, defined as the conversion efficiency versus the wavelength of the source for a fixed pump wavelength, are presented. These experimental curves result from the selection of the MIR radiation by the SFG process powered by the single-line pump laser. They are recorded by scanning the converted light with a monochromator and are very close to the theoretical squared sinc function dictated by the quasi-phase matching conditions. When the non-linear stage is fed by a flat broad-band source signal, the spectrum of the converted signal directly gives the waveguide conversion efficiency curves. The related coherence function has a triangular shape according to the Wiener-Khinchin theorem (Darré et al. 2015). The converted power $P_{\text {converted }}$ at $817 \mathrm{~nm}$ linearly depends on the signal power $P_{\text {signal }}$ at $3.5 \mu \mathrm{m}$. The measured internal conversion efficiency linearly depends on the pump power $P_{\text {pump }}$ and has a maximum slope equal to:

$$
\frac{P_{\text {converted }}}{P_{\text {signal }} \times P_{\text {pump }}}=85 \text { per cent } \mathrm{W}^{-1}
$$

when the phase-matching condition is fulfilled. The spectral acceptance bandwidth, inversely proportional to the crystal length, is experimentally equal to $\Delta \lambda_{\mathrm{s}}=37 \mathrm{~nm}$, corresponding to a converted bandwidth $\Delta \lambda_{\mathrm{c}}=2 \mathrm{~nm}$ at $817 \mathrm{~nm}$ (Lehmann et al. 2019c). This narrow spectral acceptance leads to a converted signal with a large coherence length of $280 \mu \mathrm{m}$, making it easier to find interferometric fringes, as discussed in Section 5.

In the NIR stage, the converted fields are injected in $10 \mathrm{~m}$ long polarization-maintaining single-mode fibres at the $817 \mathrm{~nm}$ operating wavelength. The fibre arms include a delay line and a phase modulator and are connected to a coupler to perform the interferometric mixing. The delay line controls the optical paths of the two arms with an accuracy much smaller than the coherence length. In the phase modulator, temporal modulation is applied by mechanical stretching of the fibre part wound around a piezoelectric ceramic driven by a linear high voltage to display the fringes as a function of time. At the outputs of the coupler, two silicon avalanche photodiodes (SiAPD), working in the photon-counting regime, detect the interferometric signals. Before the detectors, a set of optical filters removes unwanted pump residues and only lets a $20 \mathrm{~nm}$ band around $817 \mathrm{~nm}$ reach the detector.

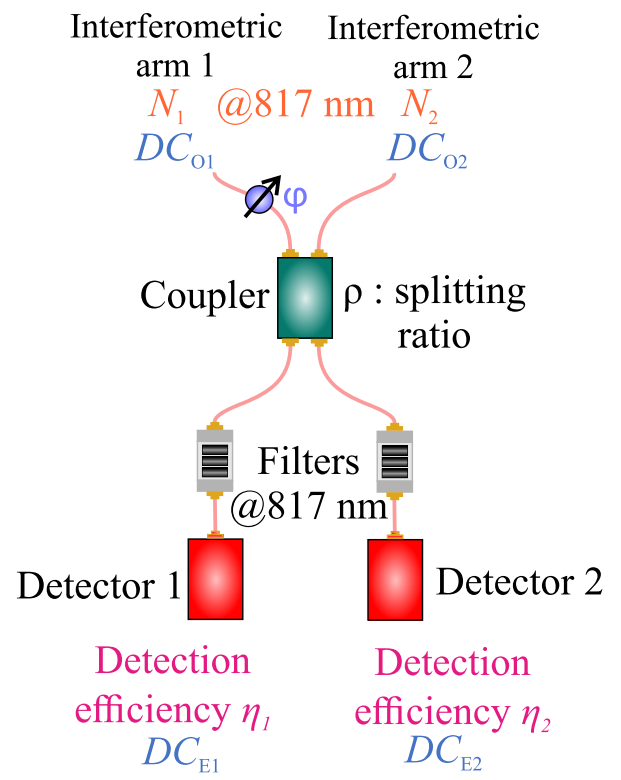

Figure 4. Diagram showing the elements of the optical bench parameters allowing us to derive equations (2) and (3). $\left\langle N_{1}\right\rangle,\left\langle N_{2}\right\rangle$ : science photon average fluxes at $817 \mathrm{~nm}$ of arms 1 and 2 respectively. $\mathrm{DC}_{\mathrm{O} 1}, \mathrm{DC}_{\mathrm{O} 2}$ : optical dark count (thermal radiation, parametric fluorescence) coming respectively from arms 1 and $2 . \mathrm{DC}_{\mathrm{E} 1}, \mathrm{DC}_{\mathrm{E} 2}$ : electrical dark count generated by detectors 1 and 2 respectively. $\varphi$ : phase modulation.

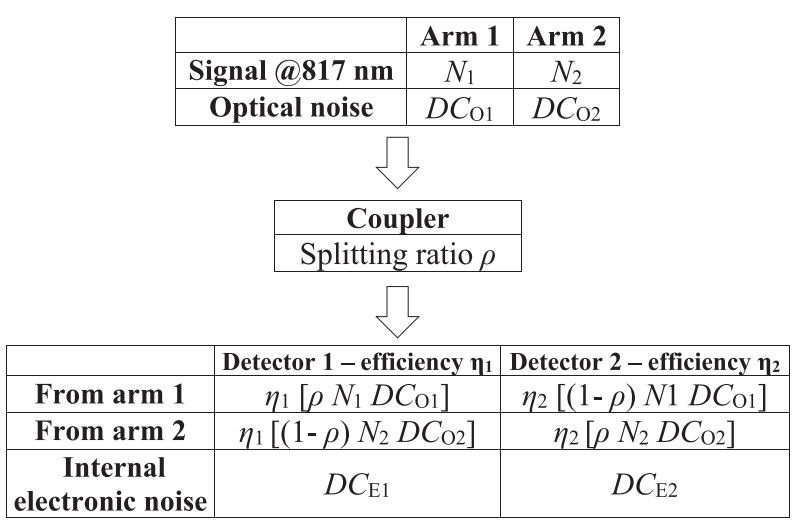

Figure 5. Table summarizing Fig. 4 in order to derive equations (2) and (3). $\left\langle N_{1}\right\rangle,\left\langle N_{2}\right\rangle$ : science photon average fluxes at $817 \mathrm{~nm}$ of arms 1 and 2 respectively. $\mathrm{DC}_{\mathrm{O} 1}, \mathrm{DC}_{\mathrm{O} 2}$ : optical dark count probability (thermal radiation, parametric fluorescence) coming respectively from arms 1 and $2 . \mathrm{DC}_{\mathrm{E} 1}$, $\mathrm{DC}_{\mathrm{E} 2}$ : electrical dark count coming respectively from detectors 1 and 2.

Figs 4 and 5 summarize the contributions involved in the derivation of the unnormalized probability density of photoevents $D_{1}$ and $D_{2}$ on the two detectors:

$$
\begin{aligned}
D_{1}= & {\left[\left\langle N_{1}\right\rangle \rho+\left\langle N_{2}\right\rangle(1-\rho)\right.} \\
& \left.+2 C_{\text {inst }} \sqrt{\left\langle N_{1}\right\rangle\left\langle N_{2}\right\rangle \rho(1-\rho)} \cos (\varphi)\right] \eta_{1}+\mathrm{DC}_{\mathrm{OE} 1} \\
D_{2}= & {\left[\left\langle N_{1}\right\rangle(1-\rho)+\left\langle N_{2}\right\rangle \rho\right.} \\
& \left.-2 C_{\text {inst }} \sqrt{\left\langle N_{1}\right\rangle\left\langle N_{2}\right\rangle \rho(1-\rho)} \cos (\varphi)\right] \eta_{2}+\mathrm{DC}_{\mathrm{OE} 2}
\end{aligned}
$$


where $\rho$ is the splitting ratio of the coupler and $\eta_{1}$ and $\eta_{2}$ the detection efficiencies of the two detectors, respectively.

$\left\langle N_{1}\right\rangle$ and $\left\langle N_{2}\right\rangle$ denote the average number of photons at $817 \mathrm{~nm}$ of each arm of the interferometer. The first three terms correspond to the flux coming from arm 1 and arm 2 and the interferometric term weighted by $C_{\text {inst }}$ (the instrumental contrast). This term results from all the instrumental defects. The last contribution, denoted $\mathrm{DC}_{\mathrm{OE} i}$ $(i=1,2)$ (optoelectronic dark count), is the global parasitic noise resulting from:

(i) parasitic signals generated by unwanted non-linear processes giving rise to radiations around $817 \mathrm{~nm}$ called $\mathrm{DC}_{\mathrm{O}}$ (optical dark count). The two major contributions are the up-conversion of the thermal background fitting the spatial mode of the non-linear crystal and the cascade of a spontaneous parametric down-conversion and an up-conversion process (Pelc et al. 2010);

(ii) the electronic dark count of the detector, called $\mathrm{DC}_{\mathrm{E}}$. The $\mathrm{DC}_{\mathrm{E}}$ of each of our detectors is equal to 100 counts per second.

The global $\mathrm{DC}_{\mathrm{OE}}$ received at each detector can be written as:

$\mathrm{DC}_{\mathrm{OE} 1}=\mathrm{DC}_{\mathrm{E} 1}+\mathrm{DC}_{\mathrm{O} 1} \rho \eta_{1}+\mathrm{DC}_{\mathrm{O} 2}(1-\rho) \eta_{1}$

$\mathrm{DC}_{\mathrm{OE} 2}=\mathrm{DC}_{\mathrm{E} 2}+\mathrm{DC}_{\mathrm{O} 1}(1-\rho) \eta_{2}+\mathrm{DC}_{\mathrm{O} 2} \rho \eta_{2}$.

These different white noises are incoherently superimposed on to the deterministic signals. In the spectral domain, the power spectral density of the deterministic signals is biased by a background offset equal to the total number of photons detected per acquisition frame. To minimize the impact of these noises, the deterministic signals are encoded on a signal spectral channel using a linear phase (i.e. optical path) modulation. In our interferometer, $\mathrm{DC}_{\mathrm{O}}$ predominates over $\mathrm{DC}_{\mathrm{E}}$ and the data processing will be designed in order to limit the impact of this noise on the fringe acquisition and contrast measurements. Notice that, at the outputs of the interferometer, the two interference patterns are $\pi$-phase shifted. This property will be taken into account using suitable linear combinations of $D_{1}$ and $D_{2}$ for efficient signal processing. After characterization of the coefficients $\rho$ and $\eta_{1} / \eta_{2}$, the calibration of the interferometer consists of three steps:

(i) the measurements of $\mathrm{DC}_{\mathrm{OE} 1}$ on detector 1 and $\mathrm{DC}_{\mathrm{OE} 2}$ on detector 2 are simultaneously performed using MIR shutters to block the two interferometric arms;

(ii) measurement of the signal $\left\langle N_{1}\right\rangle$ on the two detectors 1 and 2 simultaneously while the MIR shutter is closed on interferometric arm 2. To get $\left\langle N_{1}\right\rangle, \mathrm{DC}_{\mathrm{OE} 1}$ and $\mathrm{DC}_{\mathrm{OE} 2}$ are subtracted from the measurements on detectors 1 and 2, respectively;

(iii) in the same way, the $\left\langle N_{2}\right\rangle$ signal is measured simultaneously on both detectors 1 and 2 .

The fringe acquisition and data processing are described in Section 4.

\section{MANAGING THE THERMAL BACKGROUND AT THE INPUT OF THE INTERFEROMETER: AMPLITUDE DIVISION VERSUS WAVEFRONT DIVISION}

The goal of our in-laboratory experiment is to replicate as well as possible the future on-sky configuration to predict the expected performance during on-site observations. The classical in-laboratory way to test an interferometer is to use a Mach-Zehnder configuration. In this way, the input beamsplitter splits the light from the science source to the two interferometric arms through waveamplitude division, while the real on-sky configuration corresponds (a)

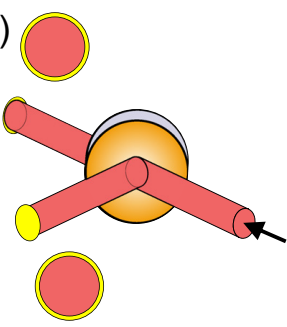

(b)

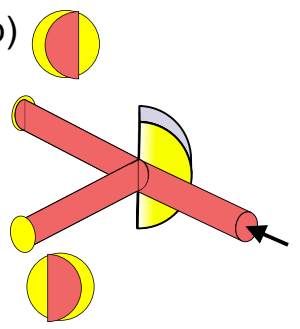

Figure 6. Diagram illustrating the overlapping of modes. The guided mode of the PPLN ridge is shown in yellow, the coupled mode with the guided mode in red. (a) Beamsplitter configuration. (b) D-shaped mirror configuration.

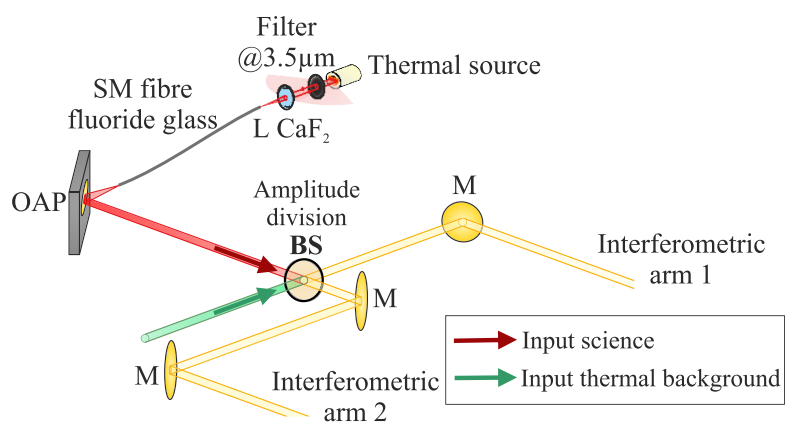

Figure 7. Beamspliter configuration: The beamsplitter shares the two input beams through amplitude division. The interference fringes generated by the main scientific source (red beam) are mixed with those generated by the thermal background (green beam). As this second input is not spatially filtered, the interference contrast is lower than that obtained with the spatially filtered scientific MIR source. This imbalance theoretically leads to an increase in contrast measured when the optical field amplitude decreases.

to wavefront division. In the visible or near-infrared this difference is not significant. In the MIR context, the results are slightly different, as emphasized below.

When using the beamsplitter method (i.e. when the optical field is divided in amplitude), the injection efficiency and the related calibration of the photometry can be mastered in each arm. In this configuration, good overlapping of the mode coming from the beamsplitter on the mode guided into the PPLN leads to quite predictable and efficient injection, as seen in Fig. 6(a). Using the blackbody radiation model for the source and evaluating the different transmission coefficients all along the propagation allow an accurate determination of the mid-infrared power coupled in each interferometric arm.

However, in such a scheme, the interferometer has an additional input source as shown in Fig. 7. The thermal background coupled via this spurious input of the beamsplitter plate generates a parasitic fringe system at a different contrast, lower than the instrumental contrast by an additional term $\Delta C$. Since the modes of the two arms of the interferometer do not match perfectly on the non-spatially filtered thermal background source, the interference contrast is biased and equal to $C_{\text {inst }}-\Delta C$. This contrast $C_{\text {biased }}$ is lower than that obtained with the science infrared optical field, spatially filtered by the singlemode fluoride glass fibre $\left(C_{\text {inst }}\right)$ :

$C_{\text {biased }}=C_{\text {inst }}+\delta C$ 
where $\delta C$ is the bias shifting the instrumental contrast depending on $\Delta C$. $C_{\text {biased }}$ can be extracted by a linear combination of the two signals detected at the output of the interferometer.

The sum of the two signals allows measurement of the global flux while the difference extracts the modulation of the interferences. The following calculations explain, in a simplified model, the impact of the second input of the beamsplitter assuming an ideal set-up with a splitting ratio of the coupler of $\rho=0.5$, with $\eta_{1}=\eta_{2}=\eta$ being the detection efficiencies of the two detectors, and $R=T=50$ percent the transmission and reflection coefficients of the beamsplitter. The exhaustive calculation, taking all the imbalance parameters of a real laboratory interferometer into account, is detailed in Appendix A.

Taking the room-temperature blackbody radiation into account, $D_{1}$ and $D_{2}$, the unnormalized probability densities of the photoevents of the two detectors, can be expressed as:

$$
\begin{aligned}
D_{1}= & \eta\left[N_{\text {exp }}\left[1+C_{\text {inst }} \cos \varphi\right]+N_{\text {room }}\left[1-\left(C_{\text {inst }}-\Delta C\right) \cos \varphi\right]\right] \\
& + \text { DC } C_{\text {OE } 1} \\
D_{2}= & \eta\left[N_{\text {exp }}\left[1-C_{\text {inst }} \cos \varphi\right]+N_{\text {room }}\left[1+\left(C_{\text {inst }}-\Delta C\right) \cos \varphi\right]\right] \\
& +\mathrm{DC}_{\mathrm{OE} 2}
\end{aligned}
$$

where $N_{\text {exp }}$ and $N_{\text {room }}$ are the photon fluxes provided by the science source and the room, respectively. $\mathrm{DC}_{\mathrm{OE} i}(i=1,2)$ (optoelectronic dark count) is the global parasitic noise introduced in Section 2.

In the following, $\left\langle X_{i 1}\right\rangle$ and $\left\langle X_{i 2}\right\rangle$ are the average values of $X_{i 1}$ and $X_{i 2}(i=1, N)$, where $X_{i 1}$ and $X_{i 2}$ are the acquisition frames related to the two interferometric outputs, tending, with high flux, towards the unnormalized probability density of photoevents $D_{1}$ and $D_{2}$ respectively. This allows us to extract the fringe modulation using the equation:

$$
\begin{aligned}
\left\langle X_{i-}\right\rangle= & \left\langle\left[X_{i 1}-X_{i 2}\right]\right\rangle \\
= & 2 \eta\left[N_{\exp }-N_{\text {room }}\right] \cos \varphi\left[C_{\text {inst }}+\Delta C \frac{N_{\text {room }}}{\left[N_{\exp }-N_{\text {room }}\right]}\right] \\
& +\mathrm{DC}_{\mathrm{OE} 1}-\mathrm{DC}_{\mathrm{OE} 2} .
\end{aligned}
$$

The resulting expression of the global contrast becomes:

$C_{\text {biased }}=C_{\text {inst }}+\delta C=C_{\text {inst }}+\frac{\Delta C}{\frac{N_{\text {exp }}}{N_{\text {room }}}-1}$.

It is important to notice that, if the influence of the second input of the beamsplitter is negligible with a bright source $\left(N_{\exp }\right.$ dominant over $N_{\text {room }}$ ), the expected contrast drifts significantly for fainter sources, as reported in the experimental results of Section 5.

For this purpose, we used a second configuration based on a Dshaped mirror (circular mirror cut in half) to perform a wavefront division of the source optical fields. In this way, the thermal backgrounds coupled in the two interferometric arms no longer generate parasitic fringes as the wavefront division ensures no spatial coherence between the two beams coming from distinct modes of the thermal background sources at $3.5 \mu \mathrm{m}$. In this configuration, our interferometer mimics the real on-sky situation where the thermal backgrounds coupled through the two telescopes are not spatially coherent.

However, as shown in Fig. 6(b), this solution is expected to have a low coupling efficiency in the interferometric arms due to the poor overlap between the guided mode of the PPLN waveguide, which is almost a circular Gaussian beam (Lehmann et al. 2019c), and the D-shaped incoming beam pattern. This results in a significant loss of the transmitted flux. Notice that the instrument also becomes more sensitive to lateral shifts of the incoming beam and the injection efficiency and the related calibration of the photometry in each arm is no longer properly controlled.

In our experimental study, these methods are used for complementary purposes in order to make our measurements. The beamsplitter configuration leads to good knowledge of the photometry in our optical bench for bright sources, while the D-shaped mirror configuration allows us to make correct contrast measurements for faint sources. The cross-interpretation of the two methods enables us to scale the flux collected in our in-laboratory experiment to a real star observation configuration. For this purpose we will use the reference flux in the $L$ band (reference in astronomy) and the blackbody Planck law (reference for the flux of our experimental blackbody) as shown in Section 5.

\section{INTERFEROMETRIC DATA PROCESSING}

Fig. 8 shows the principle of the data processing performed on our measurements. Even if the optical path difference (OPD) between the two arms of the interferometer will be servo controlled in the future on-sky configuration, random variations of the phase are expected because of various disturbances (mechanical, acoustic, thermal). The data-reduction method used to compute the fringe contrast is based on incoherent averaging, as a function of time, of the power spectral density of the recorded signals $X_{i 1}(t)$ and $X_{i 2}(t)(i=1$, $N)$ at the output of the two detectors. $X_{i 1}$ and $X_{i 2}$ are driven by the unnormalized probability density of photoevents $D_{1}$ and $D_{2}$. The generation of a photoevent either results from the scientific mid-infrared signal (coherent process) or from parasitic non-linear spurious signals (incoherently generated), as explained in Section 2. In order to discriminate these two contributions, a temporal linear OPD modulation shifts the signal on a single frequency peak while the noise is distributed uniformly over different spectral channels on the power spectral density. To extract the photometric background and the interferometric modulation, a linear combination of the $X_{i 1}$ and $X_{i 2}(i=1, N)$ signals is achieved taking into account the $\eta_{1}$ and $\eta_{2}$ coefficients and the $\pi$-phase shift between these two interferometric outputs. In the following $\tilde{X}$ will denote the Fourier transform and $|\tilde{X}(f)|^{2}$ the power spectral density of $X(t)$. With this suitable signal processing, the fringe peak at $f_{\text {mod }}$ on the power spectral density curve $\left\langle\left|\tilde{X}_{i-}\left(f_{\text {mod }}\right)\right|^{2}\right\rangle$ is typically enhanced by a factor of around four compared to the configuration using a single detector. This leads to an improvement of the signal-to-noise ratio (SNR) by a factor close to two.

The weighted sum of the temporal frames can be written as, using equations (2) and (3),

$$
\begin{aligned}
\left\langle X_{i+}\right\rangle=\left\langle\left[X_{i 1}+X_{i 2} \frac{\eta_{1}}{\eta_{2}}\right]\right\rangle= & {\left[\left\langle N_{1}\right\rangle+\left\langle N_{2}\right\rangle\right] \eta_{1} } \\
& +\mathrm{DC}_{\mathrm{OE} 1}+\mathrm{DC}_{\mathrm{OE} 2} \frac{\eta_{1}}{\eta_{2}}
\end{aligned}
$$

where $X_{i+}$ is the sum of the temporal frames $X_{i 1}$ and $X_{i 2}(i=1$, $N) .\left\langle N_{c+}\right\rangle$ is the global number of photoevents per frame and can be retrieved by averaging the power spectral density away from the zero frequency, as shown in Fig. 8.

Then, the average of the spectral density of the weighted sum at zero frequency can be written as:

$\left\langle\left|\tilde{X}_{i+}(0)\right|^{2}\right\rangle=\left\langle N_{c+}\right\rangle^{2}+\left\langle N_{c+}\right\rangle$ 


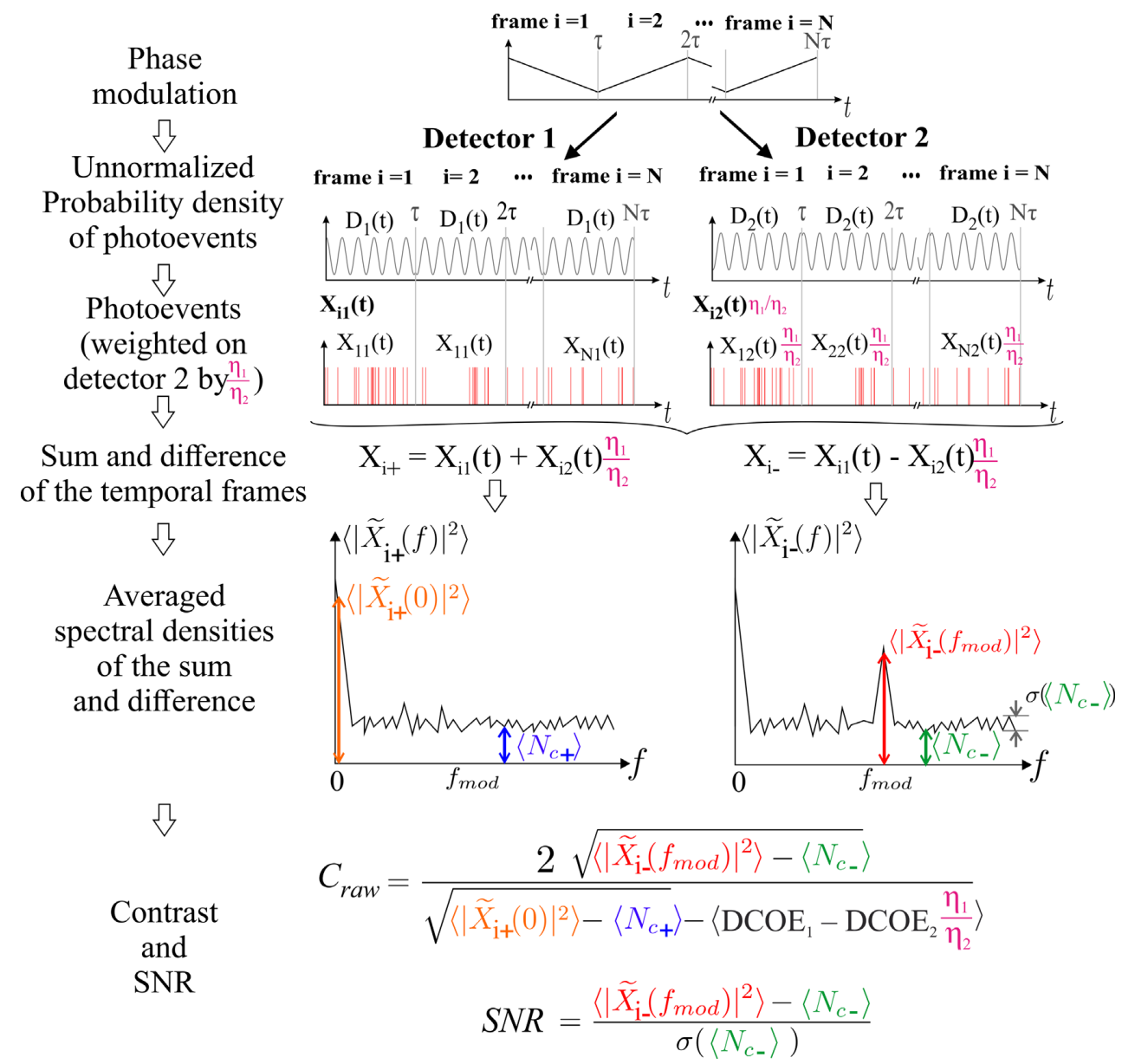

Figure 8. Data-processing method for measuring contrast and signal-to-noise ratio. $f_{\text {mod }}$ : modulation frequency, $\eta_{1}$ : efficiency of detector 1 , $\eta_{2}$ : efficiency of detector 2 .

and:

$$
\begin{aligned}
{\left[\left\langle N_{1}\right\rangle+\left\langle N_{2}\right\rangle\right] \eta_{1}=} & \sqrt{\left\langle\left|\tilde{X}_{i+}(0)\right|^{2}\right\rangle-\left\langle N_{c+}\right\rangle} \\
& -\mathrm{DC}_{\mathrm{OE} 1}-\mathrm{DC}_{\mathrm{OE} 2} \frac{\eta_{1}}{\eta_{2}}
\end{aligned}
$$

The weighted difference of the time frames can be written as:

$$
\begin{aligned}
\left\langle X_{i-}\right\rangle=\left\langle\left[X_{i 1}-X_{i 2} \frac{\eta_{1}}{\eta_{2}}\right]\right\rangle= & {\left[\left\langle N_{1}\right\rangle(2 \rho-1)+\left\langle N_{2}\right\rangle(1-2 \rho)\right] \eta_{1} } \\
& +\left[4 C_{\text {inst }} \sqrt{\left\langle N_{1}\right\rangle\left\langle N_{2}\right\rangle \rho(1-\rho)} \cos (\varphi)\right] \eta_{1} \\
& +\mathrm{DC}_{\mathrm{OE} 1}+\mathrm{DC}_{\mathrm{OE} 2} \frac{\eta_{1}}{\eta_{2}}
\end{aligned}
$$

where $\left\langle X_{i-}\right\rangle$ is the difference of the temporal frames $X_{i 1}$ and $X_{i 2}(i$ $=1, N) .\left\langle N_{c-}\right\rangle$ is the differential number of photoevents between the two detectors and can be retrieved by averaging the related power spectral density away from the modulation frequency, as shown in Fig. 8.

The spectral density of the weighted difference extracts the interferometric signal at the modulation frequency with a $C_{\text {raw }}$ contrast:

$\left\langle\left|\tilde{X}_{i-}\left(f_{\text {mod }}\right)\right|^{2}\right\rangle=\frac{1}{4} C_{\text {raw }}^{2}\left[\left\langle\left\langle N_{1}\right\rangle\right\rangle+\left\langle N_{2}\right\rangle\right]^{2} \eta_{1}^{2}+\left\langle N_{c-}\right\rangle$.
The raw contrast $C_{\text {raw }}$ is computed by substituting the terms of equation (15) with the elements of equation (13):

$C_{\mathrm{raw}}=\frac{2 \sqrt{\left\langle\left|\tilde{X}_{i-}\left(f_{\mathrm{mod}}\right)\right|^{2}\right\rangle-\left\langle N_{c-}\right\rangle}}{\sqrt{\left\langle\left|\tilde{X}_{i+}(0)\right|^{2}\right\rangle-\left\langle N_{c+}\right\rangle}-\mathrm{DC}_{\mathrm{OE} 1}-\mathrm{DC}_{\mathrm{OE} 2} \frac{\eta_{1}}{\eta_{2}}}$.

Finally, to get the instrumental contrast $C_{\text {inst }}$, the correction of the intensity imbalance is applied by using $C_{\text {phot }} \times 2 \sqrt{\rho(1-\rho)}$ :

$C_{\text {raw }}=C_{\text {inst }} 2 \sqrt{\rho(1-\rho)} \times C_{\text {phot }}$

with:

$C_{\text {phot }}=\frac{2 \sqrt{\left\langle N_{1}\right\rangle\left\langle N_{2}\right\rangle}}{\left[\left\langle N_{1}\right\rangle+\left\langle N_{2}\right\rangle\right]}$

where $C_{\text {phot }}$ results from the photometric imbalance characterized by the second and third steps of the acquisition sequence, as explained in Section 2.

As shown in Fig. 8, the signal-to-noise ratio results from the comparison between the unbiased modulation peak and the fluctuation of the noise background:

$\mathrm{SNR}=\frac{\left\langle\left|\tilde{X}_{i-}\left(f_{\mathrm{mod}}\right)\right|^{2}\right\rangle-\left\langle N_{c-}\right\rangle}{\sigma\left(\left\langle N_{c-}\right\rangle\right)}$.

The signal-to-noise ratio is the figure of merit to characterize the contrast. When we have an average SNR greater than 3, we consider that the contrast measurement is significant but potentially biased. 


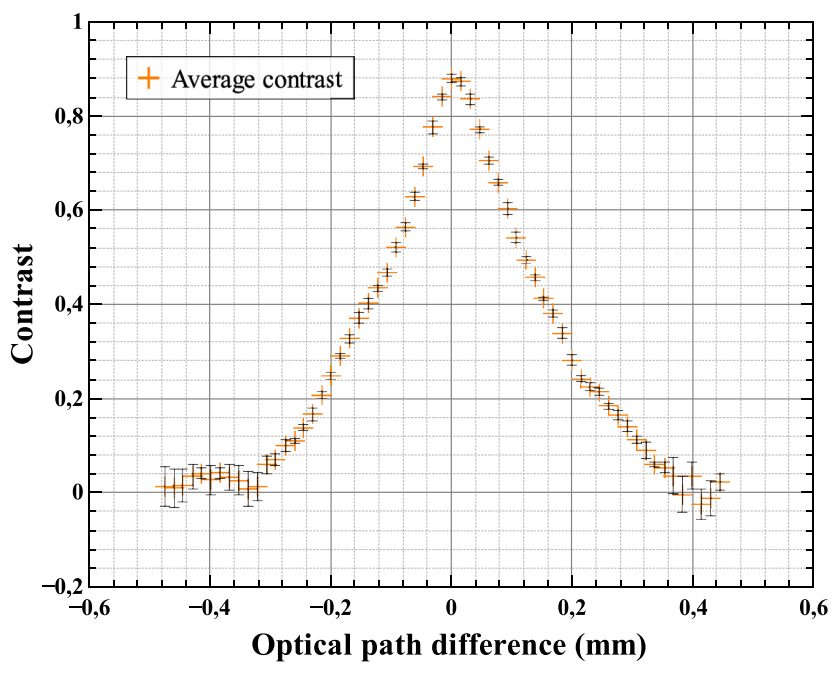

Figure 9. Visibility function of the interference fringes measured in the Dshaped mirror configuration. The number of converted photons per second during this measure was around 7700 photon s$^{-1}$ on the two arms of the interferometer. The average was calculated over six measurements. The errors bars correspond to the standard deviation of the average contrast value. All these measurements are corrected for the photometric imbalance.

\section{EXPERIMENTAL RESULTS}

In this section, the results were obtained with the D-shaped mirror configuration. In the first step, as shown in Fig. 9, the visibility function, corresponding to the contrast as a function of the optical path difference, is measured by actuating the optical delay line. The visibility function has a triangular shape due to the spectral acceptance of the PPLN, as explain in Section 2. The average values and standard deviations are calculated over six measurements, and the thermal source is set to a flux level of 7700 photons per second accumulated on the two detectors. The coherence length, characterized by the width at half maximum of the curve, is equal to $280 \mu \mathrm{m}$. The standard deviations on the contrast measurements are very low $(0.7$ per cent $\leq \sigma(C) \leq 0.9$ per cent $)$ for all measurements higher than $C=20$ per cent. For example, the average contrast $C=88$ per cent \pm 0.4 per cent with a standard deviation $\sigma(C)=$ 0.9 per cent at the maximum of the curve (zero group delay). Notice that this long coherence length and the reliability of the measurements will be a great asset for easy and accurate detection of fringes during on-sky observations.

In the second step, and after setting the interferometer to maximum contrast, the flux level of the source is varied to evaluate the reliability of the contrast measurement and the evolution of the signal-to-noise ratio as a function of the equivalent $L$ magnitude.

Fig. 10 reports the measured average contrasts with different $L$-band magnitudes (achieved by adjusting the temperature of the thermal source) obtained with the beamsplitter and D-shaped mirror configurations. As expected, the contrast bias shifts the measurement to higher values than the unbiased contrast measured with a high photon flux. Assuming a Gaussian noise statistic, the error bars correspond to the standard error on the mean value, equal to the standard deviation divided by the square root of the number of measurements. During the experiments, $\mathrm{DC}_{\mathrm{OE}}$ measurements and contrast measurements are performed alternately. Each measurement consists of 1200 acquisition frames of $0.2 \mathrm{~s}$ each sampled at $500 \mathrm{kHz}$.

As explained in Section 3, the contrast with the beamsplitter configuration drifts significantly for fainter sources. In order to

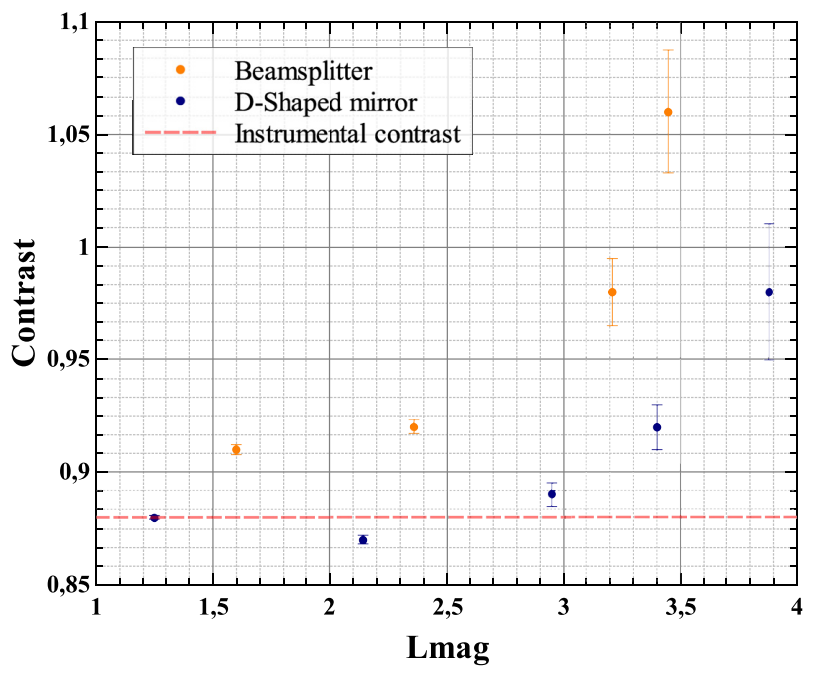

Figure 10. Contrast measurement as a function of the equivalent magnitude of the source in the $L$ band using the two configurations: beamsplitter (amplitude division) and D-shaped mirror (wavefront division). The error bars correspond to the standard error on the mean value, equal to the standard deviation divided by the square root of the number of measurements.

reduce the bias on the contrast measurement for fainter sources, it is necessary to switch from the beamsplitter to the D-shaped mirror configuration. The two sets of results are related to the photon-flux measurements $\left\langle N_{1}\right\rangle$ and $\left\langle N_{2}\right\rangle$ and plot as a function of $L_{\text {mag }}$. For each temperature of the source, the equivalent $L$ magnitude can be derived thanks to the Planck blackbody model and assuming a global experimental context including a $1 \mathrm{~m}$ telescope diameter and all the laboratory component transmission coefficients.

Table 1 reports the contrast and signal-to-noise ratio results averaged over 10 measurements for different experimental temperatures of the source. The $C=88$ per cent target value on contrast has been determined with high photon flux (i.e. very low noises). Fig. 10 and Table 1 demonstrate the meaningful reduction of the contrast bias measurement when using the D-shaped mirror configuration. The residual effect can be explained by a faint crosstalk between the two thermal backgrounds related to the two interferometric inputs. Notice that this bias will vanish during a real on-sky observation. These results show that, with this laboratory experimental context, an $L$-band magnitude of around 3.9 , equivalent to $3.0 \mathrm{fW} \mathrm{nm}^{-1}$, could be observed on-sky. In our experimental configuration, the noise is dominated by the up-conversion of the thermal background and the cascade of a spontaneous parametric down-conversion and an up-conversion process bundled in the $\mathrm{DC}_{\mathrm{O}}$ term. For example, on the 3.9 equivalent $L$ magnitude experiment, the mean $\mathrm{DC}_{\mathrm{OE}}$ value $\left(\mathrm{DC}_{\mathrm{OE} 1}+\mathrm{DC}_{\mathrm{OE} 2}\right)$ is around 4200 counts per second, while the converted signal is around 185 counts per second. The fluctuation of the $\mathrm{DC}_{\mathrm{O}}$ drives the limit of detection and the related signal-to-noise ratio.

Notice that our experiment is currently subject to many limitations. However, it could be possible to enhance and scale the sensitivity of our instrument, in comparison with MATISSE, according to the following points:

(i) The limited spectral bandwidth of the up-conversion process is $37 \mathrm{~nm}$. Our experiment uses only $37 / 100$ of the $100 \mathrm{~nm}$ MATISSE $L$ spectral bandwidth in the lower spectral resolution configuration $(R$ $=35$ ). This ratio is equivalent to a gap of $1.08 \mathrm{mag}$. 
Table 1. Results with the D-shaped mirror configuration. Mean and standard deviation over 10 measurements. The measurements were completed at room temperature $\left(20^{\circ} \mathrm{C}\right)$. Temperature: temperature of the blackbody source. Equiv $L_{\mathrm{mag}}$ : equivalent $L$-band magnitude with a $1 \mathrm{~m}$ telescope and only including our in-laboratory set-up losses. SNR: signal-to-noise ratio. All these contrast measurements are corrected from the photometric imbalance.

\begin{tabular}{lcccccc}
\hline Photon flux $\left(\mathrm{s}^{-1}\right)$ & Temperature $\left({ }^{\circ} \mathrm{C}\right)$ & Equiv $L_{\text {mag }}$ & Mean value & $\begin{array}{c}\text { Contrast } \\
\text { Standard error }\end{array}$ & Standard deviation & SNR \\
\hline 6800 & 90 & 1.25 & $88 \%$ & $0.1 \%$ & $0.2 \%$ & 2342 \\
1300 & 65.7 & 2.14 & $87 \%$ & $0.2 \%$ & $0.6 \%$ & 176 \\
540 & 45.7 & 2.95 & $89 \%$ & $0.5 \%$ & $1.7 \%$ & 38 \\
315 & 35.7 & 3.4 & $92 \%$ & $1.0 \%$ & $3.1 \%$ & 14.3 \\
185 & 25.7 & 3.88 & $98 \%$ & $3.0 \%$ & $9.6 \%$ & 5.4 \\
\hline
\end{tabular}

(ii) The non-linear components have high Fresnel reflection losses, with a 70 per cent transmission coefficient, due to the high refractive index difference between air and the PPLN. This ratio is equivalent to a gap of $0.39 \mathrm{mag}$.

(iii) The global transmission of the star simulator including the blackbody emissivity and whole transmission of the fibre filtering device is equal to 71 per cent, i.e. $0.38 \mathrm{mag}$.

The equivalent magnitude would reach 5.75 on $1 \mathrm{~m}$ telescopes assuming a $100 \mathrm{~nm}$ spectral bandwidth.

In comparison, according to the ESO website, ${ }^{1}$ in the lower spectral resolution configuration $(R=35)$, the $L$-band sensitivity limit of MATISSE in real on-sky observations with $1.8 \mathrm{~m}$ diameter telescopes is around $0.2 \mathrm{Jy}$ using the GRAVITY fringe tracker (GRA4MAT mode) equivalent to a $7.9 \mathrm{~L}$-band magnitude. This corresponds to a $6.62 \mathrm{~L}$ magnitude with $1 \mathrm{~m}$ diameter telescopes. Without the fringe tracker, the $L$-band sensitivity limit of MATISSE in real on-sky observations with $1.8 \mathrm{~m}$ diameter telescopes is around $1 \mathrm{Jy}$, equivalent to a $6.14 \mathrm{~L}$-band magnitude. This corresponds to a $4.87 L$ magnitude with $1 \mathrm{~m}$ diameter telescopes. Even if this comparison does not take into account the whole on-sky CHARA set-up losses discussed in the conclusion, this result demonstrates the potential of the ALOHA project.

\section{CONCLUSION AND PROSPECTS}

To conclude, our up-conversion interferometer prototype at $3.5 \mu \mathrm{m}$, using a wavefront division set-up, allows us to obtain fringes at $817 \mathrm{~nm}$ in laboratory conditions. The coherence length related to the selected spectrum is equal to $280 \mu \mathrm{m}$ (resolution $R$ around 100 calculated with $\lambda=3500 \mathrm{~nm}$ and $\Delta \lambda=37 \mathrm{~nm}$ ) and the contrast is 88 percent. The experimentation was performed in a photoncounting regime with a blackbody source at light levels compliant with astronomical observations. With our experimental configuration and assuming a perfect collecting telescope of $1 \mathrm{~m}$ diameter, the limiting magnitude in the $L$ band could reach $L_{\text {mag }}=3.9$, equivalent to $3.0 \mathrm{fW} \mathrm{nm}^{-1}$. These very good results allow us to consider the next step of this project, which aims to detect fringes at $3.5 \mu \mathrm{m}$ during on-sky observations at CHARA. The long coherence length and reliable measurements will be a great asset to manage the delay-line adjustment to observe fringes in on-sky tests.

In order to properly prepare the future tests on-sky at $3.5 \mu \mathrm{m}$, preliminary missions were carried out. First, a mission at the C2PU observatory of practical on-sky sensitivity tests using a single

${ }^{1}$ https://www.eso.org/sci/facilities/paranal/instruments/matisse/inst.html arm of the future ALOHA up-conversion interferometer at $3.5 \mu \mathrm{m}$ demonstrated the potential of the ALOHA project in the $L$ band (Lehmann et al. 2019b). Second, a mission at CHARA was performed to characterize and stabilize a $2 \times 200 \mathrm{~m}$ outdoor fibre at $1.55 \mu \mathrm{m}$ (Lehmann et al. 2019a) in order to correctly understand how to set up a servo control. Then, during another mission, two $200 \mathrm{~m}$ singlemode fibres at $817 \mathrm{~nm}$ to be used in our future mission were installed outdoors at CHARA.

Due to the configuration of the CHARA array, there are several differences to take into account between the future on-sky experiments and the laboratory results reported in this paper. In order to evaluate the potential of our instrument, a first sensitivity test is planned for the next mission. Nevertheless, we propose a prospective evaluation below. First, the atmospheric turbulence may impact the starlight coupling into the waveguide of the frequency conversion stages. Assuming a correlation radius $r_{0}=10 \mathrm{~cm}$ at $500 \mathrm{~nm}$, we consider $r_{0}$ around $1 \mathrm{~m}$ at $3.5 \mu \mathrm{m}$ using the relation $r_{0} \propto \lambda^{6 / 5}$. The corresponding coherence surface could be equal to the whole surface of the telescope, which leads to almost no diminution of the limit magnitude due to atmospheric turbulence. Secondly, the adaptiveoptics system (AO) collects the light with a dichroic plate to feed the wavefront sensor. Unfortunately, this component is not optimized for our operating wavelength range and the transmission at $3.5 \mu \mathrm{m}$ of this dichroic plate was measured at 30 per cent, corresponding to an attenuation of $1.3 \mathrm{mag}$. Lastly, the losses of the free-space delay lines of CHARA have to be taken into account. The transmission of the delay lines was measured at around 70 percent at $1550 \mathrm{~nm}$; we consider that we can obtain a similar transmission coefficient at the converted wavelength $(817 \mathrm{~nm})$. This corresponds to an attenuation of $0.4 \mathrm{mag}$. The losses due to the $200 \mathrm{~m}$ long optical fibres are less than $0.8 \mathrm{~dB}$, corresponding to 0.2 mag. Taking into account all of these losses leads us to anticipate the detection of fringes on stars with magnitudes in the $L$ band in the range of $L_{\text {mag }}=2.0$.

\section{ACKNOWLEDGEMENTS}

This work has been financially supported by the Centre National d'Études Spatiales (CNES), Thales Alenia Space, and the Institut National des Sciences de l'Univers (INSU).

\section{DATA AVAILABILITY}

The data underlying this article will be shared on reasonable request to the corresponding author. 


\section{REFERENCES}

Boyd R. W., 1977, Opt. Eng., 16, 166563

Brummelaar T. A. t. et al., 2005, ApJ, 628, 453

Brustlein S., Del Rio L., Tonello A., Delage L., Reynaud F., Herrmann H., Sohler W., 2008, Phys. Rev. Lett., 100, 153903

Chauvet M., Henrot F., Bassignot F., Devaux F., Gauthier-Manuel L., Pêcheur V., Maillotte H., Dahmani B., 2016, J. Opt., 18, 085503

Darré P., Szemendera L., Grossard L., Delage L., Reynaud F., 2015, Opt. Express, 23, 25450

Darré P. et al., 2016, Phys. Rev. Lett., 117, 233902

Gomes J.-T. et al., 2014, Phys. Rev. Lett., 112, 143904

Lawson P. R., Course Notes from the 1999 Michelson Summer School August 15 -19, 1999 JPL Publication 00-009 07/00, 2000, in Principles of Long Baseline Stellar Interferometry. Available at: http://adsabs.harvard.edu/a bs/2000plbs.conf.....L

Lehmann L. et al., 2018, Exp. Astron., 46, 447

Lehmann L., et al., 2019, Exp. Astron., 47, 303

Lehmann L. et al., 2019, MNRAS, 485, 3595

Lehmann L., Grossard L., Delage L., Reynaud F., Chauvet M., Bassignot F., 2019c, Opt. Express, 27, 19233

Lopez B. et al., 2014, The Messenger, 157, 8

Pelc J. S., Langrock C., Zhang Q., Fejer M. M., 2010, Opt. Lett., 35, 2804

Szemendera L., Darré P., Baudoin R., Grossard L., Delage L., Herrmann H., Silberhorn C., Reynaud F., 2016, MNRAS, 457, 3115

\section{APPENDIX A: BEAMSPLITTER CONFIGURATION: DETAILED CALCULATION OF BIASED CONTRAST}

The following calculations detail the derivation of the contrast taking into account the biases in a more general configuration with disymmetries between the different stages of the interferometer. Fig. A1 summarizes the origin of the different biases involved in the derivation of the unnormalized probability density of photoevents $D_{1}$ and $D_{2}$ on the two detectors.

Taking into account the thermal background, the unnormalized probability density of photoevents $D_{1}$ and $D_{2}$ related to the two detectors are:

$$
\begin{aligned}
D_{1}= & \eta_{1} N_{\mathrm{exp}}\left[\rho C_{1}^{\prime} R+(1-\rho) C_{2}^{\prime} T\right. \\
& \left.+2 \sqrt{\rho(1-\rho) C_{1}^{\prime} C_{2}^{\prime} R T} C_{\mathrm{inst}} \cos \varphi\right] \\
& +\eta_{1} N_{\text {room }}\left[\rho C_{1} T+(1-\rho) C_{2} R\right. \\
& -2 \sqrt{\rho(1-\rho) C_{1}^{\prime} C_{2}^{\prime} R T} C_{\mathrm{inst}} \cos \varphi \\
& \left.+2 \sqrt{\rho(1-\rho) C_{1} C_{2} R T} \Delta C \cos \varphi\right]+\mathrm{DC} \mathrm{OE} 1_{1} \\
D_{2} \times \frac{\eta_{1}}{\eta_{2}}= & \eta_{1} N_{\mathrm{exp}}\left[(1-\rho) C_{1}^{\prime} R+\rho C_{2}^{\prime} T\right. \\
& \left.-2 \sqrt{\rho(1-\rho) C_{1}^{\prime} C_{2}^{\prime} R T} C_{\mathrm{inst}} \cos \varphi\right] \\
& +\eta_{1} N_{\text {room }}\left[(1-\rho) C_{1} T+\rho C_{2} R\right. \\
& +2 \sqrt{\rho(1-\rho) C_{1}^{\prime} C_{2}^{\prime} R T} C_{\mathrm{inst}} \cos \varphi \\
& \left.-2 \sqrt{\rho(1-\rho) C_{1} C_{2} R T} \Delta C \cos \varphi\right]+\mathrm{DC} \mathrm{OE} 22_{2} \frac{\eta_{1}}{\eta_{2}}
\end{aligned}
$$

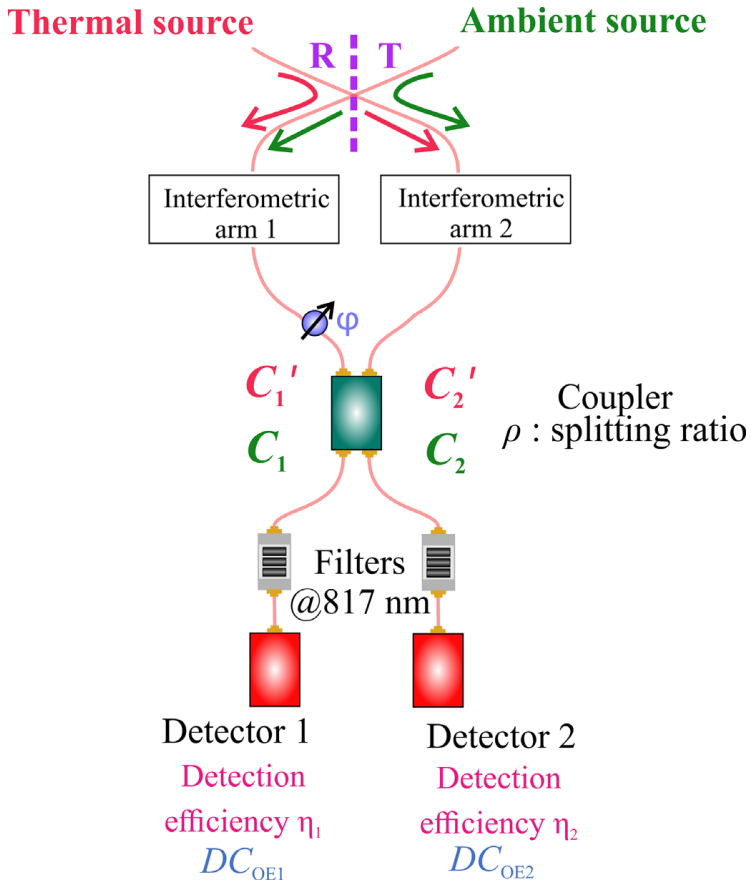

Figure A1. Diagram showing the elements of the optical bench parameters allowing us to derive equations (A1) and (A2). $C_{1}^{\prime}$ and $C_{2}^{\prime}$ : quantum efficiency of conversion, taking into account optical transmission losses, obtained with an IR source spatially filtered by a fluorinated glass fibre for the interferometric arms 1 and 2 respectively. $C_{1}$ and $C_{2}$ : quantum efficiency of conversion, taking into account optical transmission losses, obtained with a source spatially unfiltered for the interferometric arms 1 and 2 respectively. $\mathrm{DC}_{\mathrm{OE} 1}$ and $\mathrm{DC}_{\mathrm{OE} 2}$ : sum of the optical dark count (thermal radiation, parametric fluorescence) coming, respectively, from arms 1 and 2 and electrical dark count generated by detectors 1 and 2, respectively. $\varphi$ : phase modulation.

where $C_{\text {inst }}$ is the instrumental contrast, $C_{i}^{\prime}(i=1,2)$ is the quantum efficiency of the arm $i$ for an IR source spatially filtered by a fluoride glass fibre, and $C_{i}(i=1,2)$ is the quantum efficiency of the arm $i$ (conversion and transmission) for a source without spatial filtering. $R$ and $T$ denote the reflection and transmission coefficients of the beamsplitter. $\rho$ is the splitting ratio of the coupler. $\eta_{1}$ and $\eta_{2}$ are the detection efficiencies of the two detectors, respectively. $N_{\exp }$ and $N_{\text {room }}$ are the photon fluxes provided by the science source and the room, respectively. $\mathrm{DC}_{\mathrm{OE} i}(i=1,2)$ (optoelectronic dark count) is the global parasitic noise presented in Section 2.

The weighted difference of the time frames can be written as:

$$
\begin{aligned}
\left\langle X_{i-}\right\rangle=\left\langle X_{i 1}-X_{i 2} \times \frac{\eta_{1}}{\eta_{2}}\right\rangle= & \eta_{1} N_{\exp }\left[(2 \rho-1) R C_{1}^{\prime}+(1-2 \rho) T C_{2}^{\prime}\right. \\
& \left.+4 \sqrt{\rho(1-\rho) C_{1}^{\prime} C_{2}^{\prime} R T} C_{\text {inst }} \cos \varphi\right] \\
& +\eta_{1} N_{\text {room }}\left[(2 \rho-1) T C_{1}+(1-2 \rho) R C_{2}\right. \\
& -4 \sqrt{\rho(1-\rho) C_{1}^{\prime} C_{2}^{\prime} R T} C_{\text {inst }} \cos \varphi \\
& \left.+4 \sqrt{\rho(1-\rho) C_{1} C_{2} R T} \Delta C \cos \varphi\right] \\
& +\mathrm{DC}_{\mathrm{OE} 1-\mathrm{DC}_{\mathrm{OE} 2} \times \frac{\eta_{1}}{\eta_{2}}}
\end{aligned}
$$


The spectral density of the weighted difference at modulation frequency can be written as:

$$
\begin{aligned}
\left\langle\left|\tilde{X}_{i-}\left(f_{\text {mod }}\right)\right|^{2}\right\rangle= & {\left[4 C _ { \text { inst } } \eta _ { 1 } \left[N_{\exp } \sqrt{\rho(1-\rho) C_{1}^{\prime} C_{2}^{\prime} R T}\right.\right.} \\
& \left.-N_{\text {room }} \sqrt{\rho(1-\rho) C_{1} C_{2} R T}\right] \\
& \left.+4 \Delta C \eta_{1} N_{\text {room }} \sqrt{\rho(1-\rho) C_{1} C_{2} R T}\right]^{2} \\
& +\left\langle N_{c-}\right\rangle
\end{aligned}
$$

The expression of the contrast extracted from the precedent equation (A4) is:

$$
\begin{gathered}
\frac{\sqrt{\left\langle\left|\tilde{X}_{i-}\left(f_{\text {mod }}\right)\right|^{2}\right\rangle-\left\langle N_{c-}\right\rangle}}{4 \eta_{1}\left[N_{\exp } \sqrt{\rho(1-\rho) C_{1}^{\prime} C_{2}^{\prime} R T}-N_{\text {room }} \sqrt{\rho(1-\rho) C_{1} C_{2} R T}\right]} \\
=C_{\text {inst }}+\frac{\Delta C}{\frac{N_{\text {exp }}}{N_{\text {room }}} \sqrt{\frac{C_{1}^{\prime} C_{2}^{\prime}}{C_{1} C_{2}}-1}}=C_{\text {inst }}+\delta C=C_{\text {biased }} .
\end{gathered}
$$

This paper has been typeset from a $\mathrm{T}_{\mathrm{E}} \mathrm{X} / \mathrm{LT} \mathrm{E} \mathrm{X}$ file prepared by the author. 